\title{
CONTEXTO BRASILEIRO DAS PESQUISAS CIENTÍFICAS ACERCA DA SAÚDE OCUPACIONAL DO TRABALHADOR RURAL: UM LEVANTAMENTO NA BASE DE DADOS SCOPUS ELSEVIER
}

\section{Lucas Capita Quarto ${ }^{1}$}

Universidade Estadual do Norte Fluminense Darcy Ribeiro (UENF)

Daniel Madeira Cardoso²

Universidade Federal de Juiz de Fora - Campus Governador Valadares

Cristina de Fátima de Oliveira Brum Augusto de Souza ${ }^{3}$

Universidade Estadual do Norte Fluminense Darcy Ribeiro (UENF)

\begin{abstract}
Resumo
O presente trabalho tem como objetivo responder ao seguinte questionamento: quais as características das pesquisas científicas brasileiras a respeito da saúde ocupacional do trabalhador rural? Para tanto, foi realizado um levantamento na base de dados Scopus Elsevier, do periódico CAPES, no dia 10 de março de 2021, com os termos de busca: "RURAL AND WORKER AND HEALTH AND OCCUPATIONAL". Como resultado, obteve-se 128 documentos. O ano com maior índice de pesquisas foi 2.019; a medicina se destaca no que concerne os campos das ciências; em relação ao periódico, a Revista Ciência e Saúde Coletiva é o primeiro lugar no ranking; os autores com maior número de pesquisas são Moreira, J. C. e Cezar-Vaz, R. M.; o trabalho brasileiro citado é intitulado: "Influência de fatores socioeconômicos sobre o envenenamento por pesticida, Brasil"; no que tange as agências de fomento, destaca-se o Conselho Nacional de Desenvolvimento Científico e Tecnológico; o artigo científico é a modalidade de divulgação mais utilizada no Brasil para disseminar as pesquisas científicas a respeito da saúde do trabalhador rural. O presente estudo salienta a necessidade das pesquisas brasileiras se aterem às intervenções que precisam ser realizadas na esfera da saúde ocupacional do trabalhador rural.

Palavras-chave: Saúde ocupacional. Trabalhador rural. Bibliometria.

\section{Brazilian context of scientific research on occupational health of rural workers: a survey based on data Scopus Elsevier}

\begin{abstract}
This paper aims to answer the following question: what are the characteristics of Brazilian scientific research regarding the occupational health of rural workers? To this end, a survey was carried out in the database Scopus Elsevier, of the CAPES journal, on March 10, 2021, with the search terms: "RURAL AND WORKER AND HEALTH AND OCCUPATIONAL". As a result, 128 documents were obtained. The year with the highest research index was 2,019; medicine stands out with regard to the fields of science; in relation to the journal, Revista Ciência e Saúde Coletiva is the first place in the ranking; the authors with the most research are Moreira, J. C. and Cezar-Vaz, R. M .; the aforementioned Brazilian work is entitled: "Influence of socioeconomic factors on pesticiina poisoning, Brazil"; with regard to development agencies, the National Council for Scientific and Technological Development stands out; the scientific article is the most widely used type of dissemination in Brazil to disseminate scientific research on the health of rural workers. The present study highlights the need for Brazilian research to stick to interventions that need to be carried out in the field of occupational health of rural workers.
\end{abstract}

Keywords: Occupational health. Rural worker. Bibliometrics.

\footnotetext{
${ }^{1}$ Mestrando em Cognição e Linguagem na Universidade Estadual do Norte Fluminense Darcy Ribeiro (UENF). Campos dos Goytacazes, Rio de Janeiro, Brasil. Avenida Alberto Lamego, 2000, Parque Califórnia, Campos dos Goytacazes, RJ, Brasil, CEP: 28013-602. ORCID: https://orcid.org/0000-0003-4197-4998 E-mail: Icapitaiv@gmail.com

${ }^{2}$ Discente do curso de medicina. Universidade Federal de Juiz de Fora - Campus Governador Valadares, Brasil. E-mail: danielmadeira9@hotmail.com

${ }^{3}$ Doutoranda em Cognição e Linguagem na Universidade Estadual do Norte Fluminense Darcy Ribeiro (UENF). Campos dos Goytacazes, Rio de Janeiro, Brasil. Avenida Alberto Lamego, 2000, Parque Califórnia, Campos dos Goytacazes, RJ, Brasil, CEP: 28013-602. ORCID: https://orcid.org/0000-0002-6897-7200. E-mail: crisfbrum@gmail.com.
} 


\section{Contexto brasileño de la investigación científica sobre salud ocupacional de los trabajadores rurales: una encuesta en la base de datos Scopus Elsevier}

\section{Resumen}

Este artículo tiene como objetivo responder a la siguiente pregunta: ¿Cuáles son las características de la investigación científica brasileña sobre la salud ocupacional de los trabajadores rurales? Para ello, se realizó una encuesta en la base de datos Scopus Elsevier, de la revista CAPES, el 10 de marzo de 2021, con los términos de búsqueda: "RURAL Y TRABAJADOR Y SALUD Y OCUPACIONAL". Como resultado, se obtuvieron 128 documentos. El año con mayor índice de investigación fue el 2.019; la medicina se destaca en los campos de la ciencia; en relación con la revista, Revista Ciência e Saúde Coletiva ocupa el primer lugar en el ranking; los autores con más investigaciones son Moreira, J. C. y Cezar-Vaz, R. M.; el trabajo brasileño antes mencionado se titula: "Influencia de los factores socioeconómicos en la intoxicación por plaguicinas, Brasil"; en cuanto a las agencias de desarrollo, se destaca el Consejo Nacional de Desarrollo Científico y Tecnológico; el artículo científico es el tipo de difusión más utilizado en Brasil para difundir investigaciones científicas sobre la salud de los trabajadores rurales. El presente estudio destaca la necesidad de que la investigación brasileña se ciña a las intervenciones que deben realizarse en el ámbito de la salud ocupacional de los trabajadores rurales.

Palabras clave: Salud ocupacional. Trabajador rural. Bibliometría.

\section{INTRODUÇÃO}

As atividades econômicas relacionadas ao campo têm raízes com a história brasileira. Apesar do intenso processo de industrialização e migração rural-urbana que o Brasil passou a partir de meados dos anos 40 do século passado, ano após ano, o agronegócio brasileiro se mantém como um dos setores mais importantes do Produto Interno Bruno (PIB) do país. De acordo com os dados divulgados pelo Censo Agropecuário, as exportações do agronegócio no Brasil atingiram US\$10 bilhões em julho de 2020, o maior valor para o mês desde o início da série histórica em 1997. Contudo, o sucesso nos indicadores econômicos não reflete nos indicadores das condições de trabalho e saúde dos trabalhadores rurais.

O ramo da atividade agrícola é um dos que mais acometem a saúde e segurança dos trabalhadores, com evidentes aumentos nos índices de acidentes, lesões e doenças relacionados ao trabalho (MARTINS; FERREIRA, 2015). Durante a execução de suas atividades, o trabalhador rural está exposto a uma série de riscos de acidentes e agravos a saúde, como doenças ocupacionais e intoxicações (MOREIRA et al., 2015). De acordo com a Organização Internacional do Trabalho (OIT), a agricultura se encontra inclusa no grupo de atividades econômicas com maior número de óbitos entre os colaboradores (BEVILAQUA et al., 2020). A diferença entre o trabalho rural e as demais atividades são características que culminam nas condições de saúde e segurança, como a extensa jornada longa de trabalho, a sazonalidade, o forço físico, o caráter cíclico, a exposição a mudanças climáticas e o uso indiscriminado de defensores agrícolas (MARTINS; FERREIRA, 2015).

De acordo com Moreira et al. (2015) as pesquisas no Brasil a respeito das condições de saúde dos trabalhadores rurais são escassas. Ainda segundo os autores, em relação à saúde da população rural, os estudos existentes, em sua maioria, são destinados a exposições ou morbidades específicas, como ruídos, agrotóxicos, acidentes laborais e doenças respiratórias. 0 desenvolvimento de pesquisas a respeito da saúde do trabalhador rural pode subsidiar o planejamento de cuidados à saúde dessa parcela da população economicamente ativa do Brasil, o que torna evidente a necessidade da continuidade no desenvolvimento de estudos a respeito dessa temática.

Dada a importância do tema, esta pesquisa busca contribuir com a abrangência da temática ao responder a seguinte problemática: quais as características das pesquisas científicas brasileiras a respeito da saúde ocupacional do trabalhador rural? Trata-se de um levantamento bibliométrico, exploratório, que forma um cenário a partir dos autores com maior número de publicações 
[i] | contexto brasileiro das pesquisas científicas acerca da saúde ocupacional do trabalhador rural...

envolvendo os termos saúde do trabalhador rural, revistas com maior incidência do termo, áreas com maior volume de pesquisas, modalidade de divulgação, documentos mais citados, instituições que mais financiaram estudos acerca do assunto e as principais agências de fomento.

\section{METODOLOGIA}

O presente estudo se caracteriza como uma pesquisa de cunho exploratório, que tem por finalidade analisar e caracterizar a produção científica acerca da saúde ocupacional do trabalhador rural, por intermédio da bibliometria. Para tanto, foi realizado um levantamento na base de dados Scopus Elsevier, do periódico CAPES, no dia 10 de março de 2021, com os termos de busca: "RURAL AND WORKER AND HEALTH AND OCCUPATIONAL".

A plataforma Scopus retornou 1.526 documentos a nível mundial. Destarte, foi realizada um refinamento da busca, selecionando o contexto brasileiro (LIMIT-TO (AFFILCOUNTRY, "BRAZIL")) (Figura 01). O refinamento deu um retorno de 128 documentos, ou seja, o contexto brasileiro das pesquisas científicas sobre a saúde ocupacional do trabalhador rural consiste em 128 estudos. Esta análise bibliométrica abordou as variáveis: Source (Fonte), Author (Autor), Affilation (Instituição), Document type (Tipo de documento), Documents by funding sponsor (Agência de financiamento) e Subject área (Área do conhecimento).

Figura 01 - Busca na base Scopus com os descritores utilizados na pesquisa

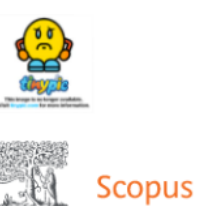

Search Sources Lists SciVal 7

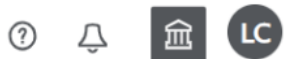

\section{Start exploring}
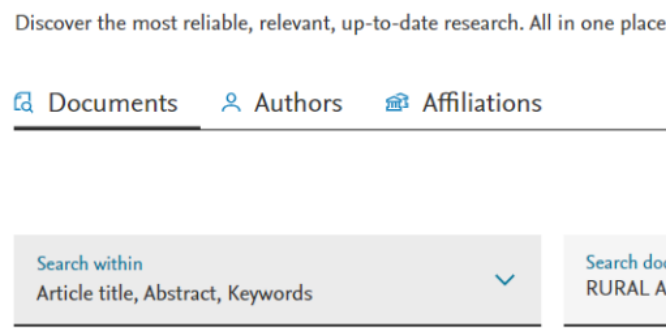

RURAL AND WORKER AND HEALTH AND OCCUPATIONAL

Fonte: Relatório de bibliometria da base Scopus (2021)

A bibliometria é um campo de estudo que permite a aplicação de métodos estatísticos e matemáticos para analisar e construir indicadores de avaliação dos aspectos quantitativos da produção e disseminação do conhecimento científico (RIBEIRO, 2017). A relação existente entre o conhecimento e a pesquisa remete aos primórdios da humanidade, no período o qual Aristóteles afirmava que novas ideais surgem através da contradição e contraposição de ideias anteriores (CHUEKE; AMATUCCI, 2015). Tal concepção salienta o fato de que devemos ter ciência do que está sendo desenvolvido acerca de um tema para evoluirmos em termos de conhecimento. Os estudos bibliométricos podem contribuir com a sistematização dos estudos realizados em um determinado 
[i.1.1] Lucas Capita Quarto, Daniel Madeira Cardoso, Cristina de Fátima de Oliveira Brum Augusto de Souza campo de saber e endereçar problemáticas que podem ser investigadas em futuras pesquisas (CHUEKE; AMATUCCI, 2015).

\section{RESULTADOS E DISCUSSÃO}

\section{Análise das pesquisas no decorrer dos anos}

Analisando os dados fornecidos pela base de dados Scopus Elsevier, por meio do recurso de análise dos resultados da pesquisa (Analyze search results), verifica-se que o primeiro estudo a respeito da temática foi registrado no ano de 1.990 e que o pico de divulgação científica ocorreu em 2.019 (Figura 02). A imagem também permite aferir uma variação no quantitativo de publicações no decorrer dos anos.

Figura 02 - Documentos por ano

\section{Documentos por ano}

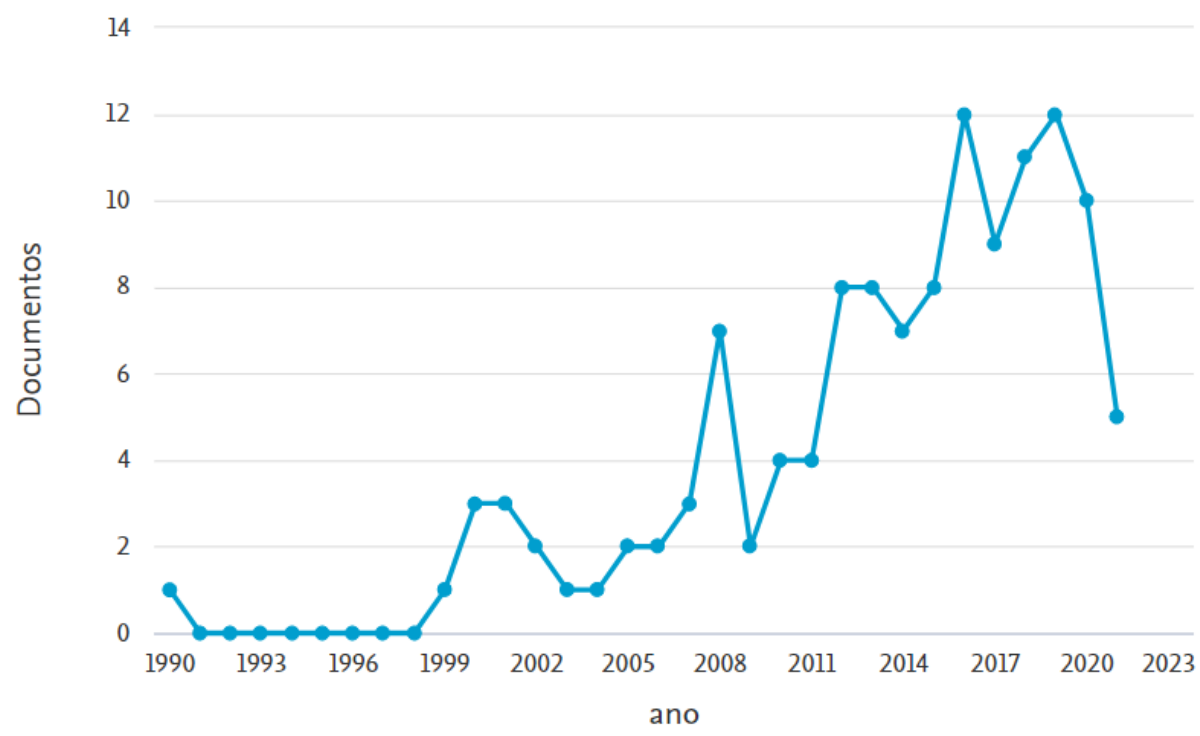

Fonte: Relatório de bibliometria da base Scopus (2021)

O quantitativo de manuscritos publicados, bem como a representação gráfica da Figura 02 revelam que, apesar do baixo número de pesquisas sobre o assunto, esta temática está sendo cada vez mais divulgada no cenário científico. Entretanto, o fato do Brasil ser um dos maiores exportadores do agronegócio do mundo e de possuir um considerável percentual de sua população concentrada na área rural, torna necessário a ampliação desse campo de estudo.

\section{Análise dos documentos por área}

A bibliometria é uma ferramenta que auxilia na identificação de tendências de progressão do conhecimento, assuntos relevantes ou obsoletos e dispersões (RIBEIRO, 2017). Nesse cenário, no que concerne os campos das ciências, destacam-se: a medicina (89 documentos; 49,7\%); as ciências ambientais (16; 8,9\%); e as ciências agrícolas e biológicas (11; 6,1\%) (Figura 03). 


\section{Figura 03 - Documentos por área de assunto}

Documentos por área de assunto

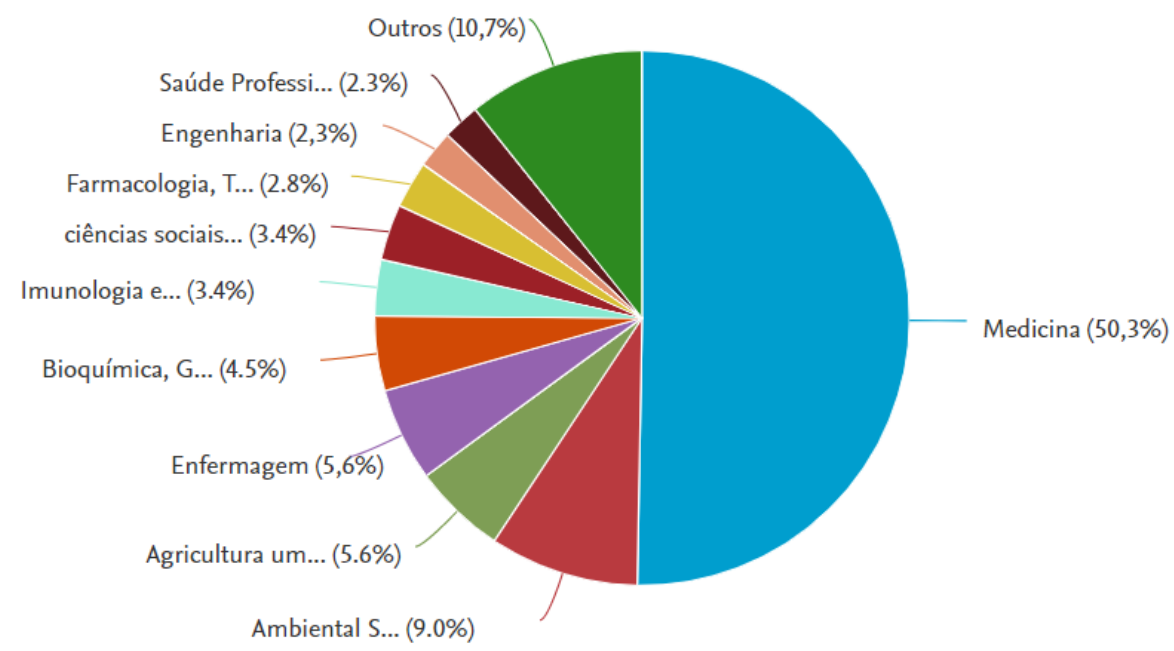

Fonte: Relatório de bibliometria da base Scopus (2021)

A medicina, primeiro lugar no ranking, é um campo do saber fundamental no desenvolvimento de estudos a respeito da saúde ocupacional no contexto geral. É pertinente destacar que um mesmo documento pode apresentar uma abordagem interdisciplinar, multidisciplinar ou pluridisciplinar ou seja, pode ser vinculado a mais de uma área do conhecimento.

\section{Análise dos documentos por fonte}

Em sequência, conforme a proposta do estudo, o Gráfico da Figura 04, realiza um cruzamento entre fontes acadêmicas, conservando os 128 documentos. Como resultado, destacamse os periódicos: Revista Ciência e Saúde Coletiva, Revista de Saúde Pública e Cadernos de Saúde Pública (Figura 04). 


\section{Documents per year by source}

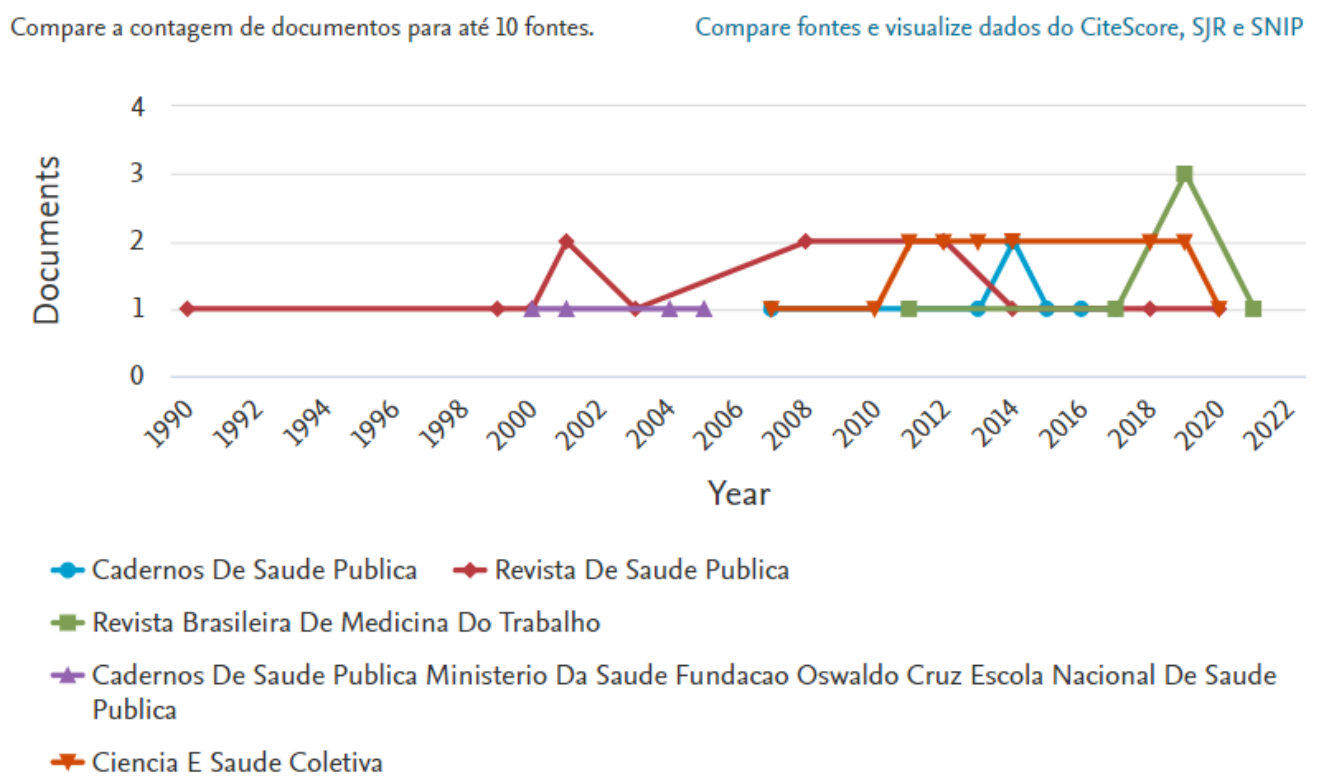

Fonte: Relatório de bibliometria da base Scopus (2021)

Criado em 1996, o periódico "Revista Ciência e Saúde Coletiva" é um espaço para debates, discussões, exposição de pesquisas e controvérsias a respeito das subáreas da área da saúde, com a categoria A3 no Qualis/Capes (CSC, 2021). Vinculada a Universidade de São Paulo (USP), a Revista de Saúde Pública tem cinquenta anos de dedicação ao campo da saúde pública, sendo referência em âmbito nacional e internacional (RSP, 2021). O Caderno de Saúde Pública se dedica a divulgação de pesquisas relacionadas a saúde ambiental, nutrição, políticas públicas, ciências sociais aplicadas à saúde, ecologia, parasitologia e epidemiologia (CSP, 2021).

\section{Análise dos documentos por autor}

Na concepção de Quarto et al. (2020) um dos pilares da bibliometria é a identificação e análise dos autores mais produtivos sobre um determinado tema. $\mathrm{O}$ autor ainda destaca que aprofundar em respectivas abordagens pode subsidiar na compreensão de diferentes pontos de vistas, assim como em novas realidade e conhecimentos. A Figura 05 demonstra destaque para os autores Moreira, J. C. e Cezar-Vaz, R. M. 


\section{Documentos por autor \\ Compare a contagem de documentos para até 15 autores.}

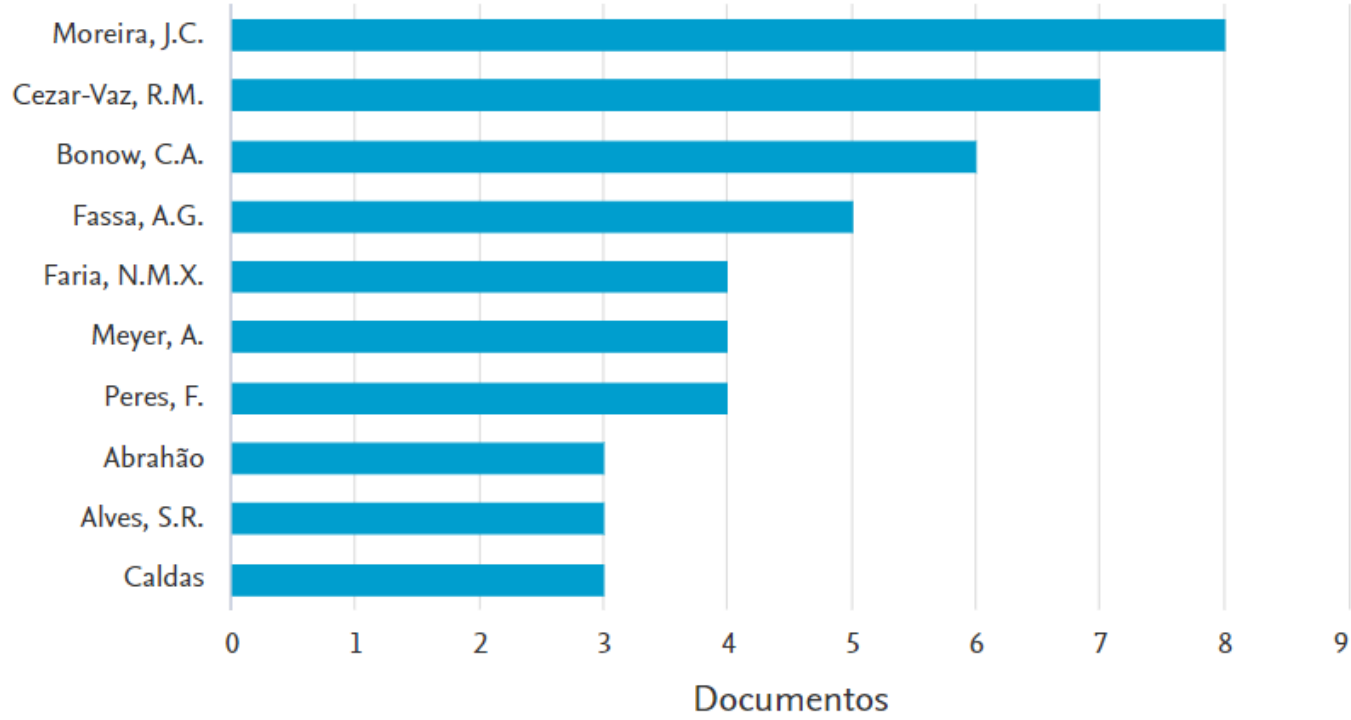

Fonte: Relatório de bibliometria da base Scopus (2021)

O manuscrito mais recente de Moreira, J. C., relacionado a temática desta pesquisa, trata-se de um artigo científico intitulado "Doença do Tabaco Verde entre trabalhadores rurais brasileiros e polimorfismos genéticos". O objetivo desta pesquisa é determinar a prevalência da doença do tabaco verde em uma população de trabalhadores rurais de uma área produtora localizada no Nordeste brasileiro e investigar se a patologia está associada a fatores como sexo, idade e estado de tabagismo. Para tanto, foram aplicados questionários: sociodemográfico, comportamental e ocupacional. Como resultado, obteve-se uma prevalência total da doença de tabaco verde em 56,9\% da amostra, com uma diferente significativa entre os gêneros $(71,7 \%$ para as mulheres e $35,3 \%$ para os homens, $p<0,0001)$. Como conclusão, a investigação salienta a necessidade da implementação de medidas preventivas para proteger os trabalhadores rurais.

A pesquisa mais recente de Cezar-Vaz, R. M., intitulada "Acidentes de trabalho com crianças e jovens em ambiente rural no Sul do Brasil", tem como objetivo conhecer a prevalência de acidente ocupacional em crianças e jovens que trabalham em atividades agrícolas para, posteriormente, identificar os fatores associados às moléstias. Este trabalho se classifica como um estudo exploratório, analítico e descritivo com abordagem quantitativa. Participaram da pesquisa 211 crianças e jovens em situação de trabalho rural. A coleta de dados foi realizada por meio de questionário semiestruturado. A análise bivariada foi realizada utilizando-se os testes qui-quadrado de Pearson, o exato de Fisher, os testes t e mann-whitney de Student e Mann-Whitney e a análise multivariada usando a regressão de Poisson. A prevalência de acidente ocupacional entre a amostra foi de $55 \%$. Com destaque para: picadas de insetos (44\%), queimaduras $(40,5 \%)$, quedas $(27,6 \%)$, lesão com ferramenta de trabalho $(16,4 \%)$, choque elétrico $(15,5 \%)$, queimadura por animal $(8,6 \%)$, 
II I Lucas Capita Quarto, Daniel Madeira Cardoso, Cristina de Fátima de Oliveira Brum Augusto de Souza mordida de animal (6,9\%) e intoxicação por pesticidas (2,6\%). Acredita-se que esse resultado pode potencializar o desenvolvimento de políticas públicas voltadas à preservação da saúde dessa população, regular as condições do trabalho rural e reduzir os riscos ocupacionais no ambiente rural.

As publicações científicas, independentemente do banco de dados, podem ser ordenadas por relevância, a partir da quantidade de citações dentro daquele indexador. Baseando-se em Oliveira et al. (2019), de modo a qualificar esta pesquisa, buscou-se ampliar os resultados obtidos na bibliometria, justificando os resultados obtidos na base de dados Scopus Elsevier com os dados presentes no Google Acadêmico. Com isso, os resultados encontrados na Scopus foram relacionados com os resultados da busca do Google Acadêmico corroborando a convergência dos autores com maior número de pesquisas acerca do assunto.

No que concerne o cruzamento dos dados obtidos entre a base de dados mencionada e o Google Acadêmico, a Figura 06, aponta que é possível analisar no retorno da busca feita ao autor Moreira, J. C.

Figura 06 - Retorno de busca do autor Moreira, J. C.

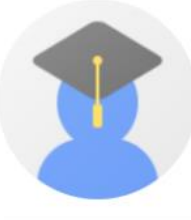

Josino Costa Moreira

Pesquisador da Escola Nacional de Saude Publica E-mail confirmado em fiocruz.br

quimica analitica biomarcadores saude ambiental
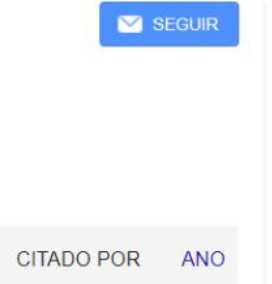

$361 \quad 2002$

Avaliação integrada do impacto do uso de agrotóxicos sobre a saúde humana em uma comunidade agrícola de Nova Friburgo, RJ

JC Moreira, SC Jacob, F Peres, JS Lima, A Meyer, JJ Oliveira-Silva,

Ciência \& Saúde Coletiva 7 (2), 299-31

Studies on the mechanisms of lead immobilization by hydroxyapatite

E Mavropoulos, AM Rossi, AM Costa, CAC Perez, JC Moreira,

Environmental science \& technology 36 (7), 1625-1629

Avaliação da contaminação humana por hidrocarbonetos policíclicos aromáticos

(HPAs) e seus derivados nitrados (NHPAs): uma revisão metodológica

AD Pereira Netto, JC Moreira, AEXO Dias, G Arbilla, LFV Ferreira.

Quimica nova 23 (6), 765-773

Fonte: Relatório de bibliometria da base Google Acadêmico

Os dados quantitativos desse autor confirmam a capilaridade e a relevância do autor, comprovando os resultados obtidos na base de dados Scopus Elsevier. O autor Cezar-Vaz, R. M., por sua vez, não possui perfil no Google Acadêmico. Ainda conforme Oliveira et al. (2019), visando possibilitar a descrição dos resultados encontrados de forma mais amplificada, a Figura 07 expressa uma compilação da estatística descritiva adotada nesse estudo bibliométrico, apontando as tendências de documentos e citações ao longo do tempo. Esses números fazem parte dos dados de domínio da base de dados Scopus Elsevier.

Ainda conforme Oliveira et al. (2019), visando possibilitar a descrição dos resultados encontrados de forma mais amplificada, a Figura 07 expressa uma compilação da estatística descritiva adotada nesse estudo bibliométrico, apontando as tendências de documentos e citações ao longo do tempo. Esses números fazem parte dos dados de domínio da base de dados Scopus Elsevier. 
[i] | Contexto brasileiro das pesquisas cientificas acerca da saúde ocupacional do trabalhador rural...

Figura 07 - As tendências de documentos e citações ao longo do tempo

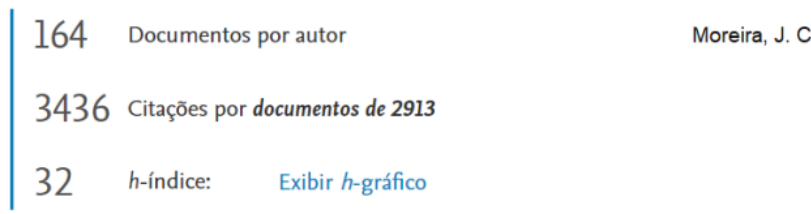

Tendências de documentos e citação
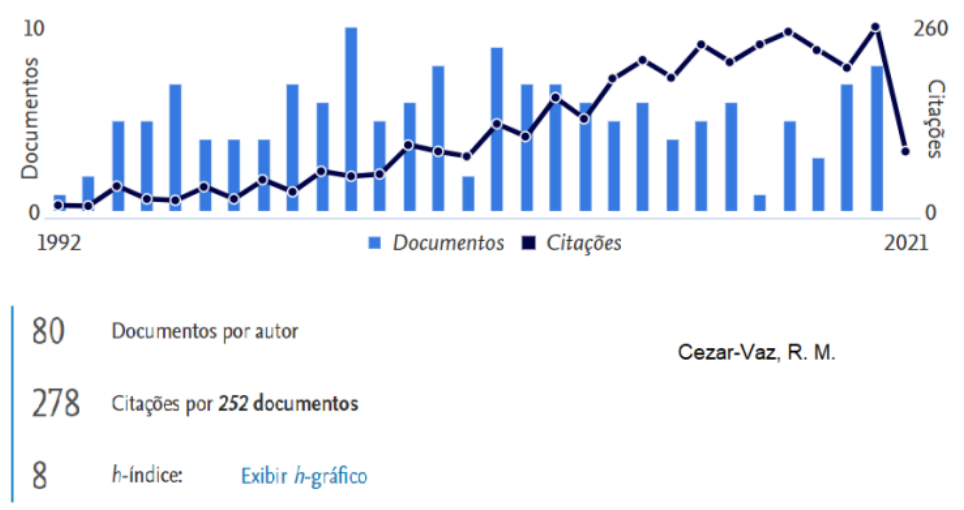

Tendências de documentos e citação

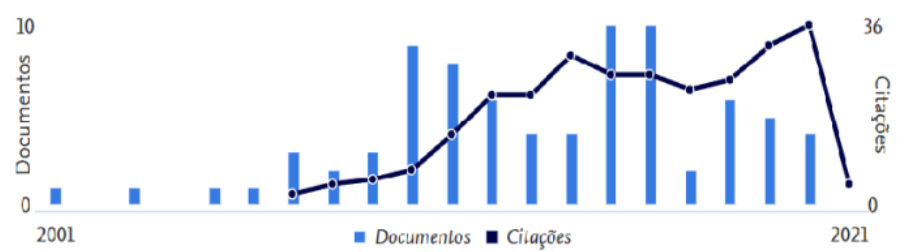

Fonte: Relatório de bibliometria da base Scopus (2021)

A Figura 7 se refere aos resultados já expostos, reforçando as características relacionadas a progressão das pesquisas científicas quanto aos autores mais produtivos no que diz respeito a temática. A imagem ainda destaca os anos em que os autores foram mais citados em trabalhos científicos.

\section{Análise dos documentos mais citados}

A bibliometria ainda permite o fichamento dos documentos mais citados a respeito de uma problemática, conforme demonstra a Figura 08, que descreve os três artigos mais mencionados em pesquisas a respeito da saúde ocupacional do trabalhador rural. 
i.j| Lucas Capita Quarto, Daniel Madeira Cardoso, Cristina de Fátima de Oliveira Brum Augusto de Souza

Figura 08 - Os documentos mais citados a respeito da problemática saúde ocupacional do trabalhador rural

Influence of social-economic factors on the pesticine
poisoning, Brazil | [Influência de fatores socioeconômicos na
contaminação por agrotóxicos, Brasil]
Open Access
Oliveira-Silva, J.J.,

Alves, S.R., Meyer,

A., (...), Da Costa

Mattos, R.D.C.O.,

Moreira, J.C.
2001 Revista de Saude

Publica

35(2), pp. 130-135
56

De Araújo, A.J., De

Lima, J.S., Moreira,

J.C., (...), Das

Neves, C.,

Markowitz, S.
2007 Ciencia e Saude

Coletiva

12(1), pp. $115-130$

efeitos à saúde: Estudo transversal em amostra de 102

trabalhadores rurais, Nova Friburgo, RJ]

Open Access

View abstract $\vee$ View at Publisher Related documents

Riquinho, D.L., Hennington, E.A.

2012 Ciencia e Saude Coletiva 17(6), pp. 1587-1600 cultivation: A review of the literature | [Saúde, ambiente condições de trabalho no cultivo de tabaco: Revisão de literature]

Open Access

View abstract $\vee$ View at Publisher Related documents

Fonte: Relatório de bibliometria da base Scopus (2021)

O Artigo com maior número de citações na base de dados Scopus tem como título "Influência de fatores socioeconômicos sobre o envenenamento por pesticiina, Brasil", tendo sido citado em 55 documentos. O objetivo desta pesquisa foi avaliar as exposições dos trabalhadores rurais do Estado do Rio de Janeiro aos pesticidas anticolinesterasicos, por meio da análise dos níveis de acetilcolinesterase de células vermelhas (AChE) e butyrilcholinesterase plasmática (BChE). Buscouse também analisar se os fatores socioeconômicos, como o nível de escolaridade, podem influenciar fortemente essa situação. Participaram uma amostra de 55 trabalhadores de cinco comunidades do município de Magé-RJ. Avaliou-se as atividades enzimáticas conforme o método ellman modificado por Oliveira-Silva. Aplicou-se um questionário sociodemográfico. A contaminação foi estimada por técnicas estatísticas multivariadas. Os dados demonstraram resultados distintos quanto à incidência de exposição excessiva de acordo com o indicador enzimático utilizado. Encontrou-se na amostra estudada, 3,0 \% para os valores BChE e 41,8 \%, segundo a AChE. Quando esses dados foram comparados aos fatores de uso socioeconômico e de agrotóxicos, mostrou-se a importância do nível educacional na prevalência de intoxicação.

Citado em 48 estudos, o segundo manuscrito com mais citações a respeito da problemática é intitulado "Exposição múltipla a pesticidas e impactos em saúde: Estudo transversal de 102 trabalhadores rurais, Nova Friburgo, Estado do Rio de Janeiro, Brasil" é uma pesquisa transversal realizada em uma comunidade agrícola da cidade de Nova Friburgo-RJ, Brasil, com o intuito de examinar os aspectos epidemiológicos, laboratoriais e clínicos da exposição múltipla a defensivos agrícolas em uma amostra de 102 pequenos agricultores. Tanto o sexo masculino quanto o feminino foram submetidos a um protocolo extensivo que incluiu um questionário de ocupação, coleta de amostras biológicas para análise toxicológica e avaliação clínica - geral e neurológica. Os resultados 
[i] | contexto brasileiro das pesquisas científicas acerca da saúde ocupacional do trabalhador rural... da pesquisa mostraram episódios leves e moderados de intoxicação aguda por organofosofatos. 0 que reforça a importância de monitoração da exposição dos trabalhadores ruais aos pesticidas.

O artigo científico "Saúde, meio ambiente e condições de trabalho no cultivo do tabaco: uma revisão de literatura", terceiro lugar no ranking (citado 36 vezes), tem como objetivo apresentar um panorama da literatura publicada entre 1.979 e 2.010 a respeito da saúde e condições de trabalho no cultivo do tabaco, com ênfase no contexto brasileiro. Para tanto, foi feito um levantamento nas bases de dados PubMed, Scopus, WilsonWeb e Bireme/OPAS, com os termos de pesquisa: tabaco, saúde agrícola, trabalhador rural, condições de trabalho, condições de trabalho inseguras e risco ocupacional. Foram analisados 37 artigos de um total de 214 identificados. Entre os efeitos descritos na literatura, destacam-se a "doença do tabaco verde", os distúrbios respiratórios e o impacto ambiental negativo.

\section{Análise dos documentos por patrocinador de financiamento}

No que tange as agências de fomento (Figura 09), destacam-se o Conselho Nacional de Desenvolvimento Científico e Tecnológico, a Coordenação de Aperfeiçoamento de Pessoal de Nível Superior, Fundação de Amparo à Pesquisa do Estado de São Paulo, Conselho Nacional de Desenvolvimento Científico e Tecnológica e a Fundação de Amparo à Pesquisa do Estado de Minas Gerais.

Figura 09 - Documentos por agência de fomento

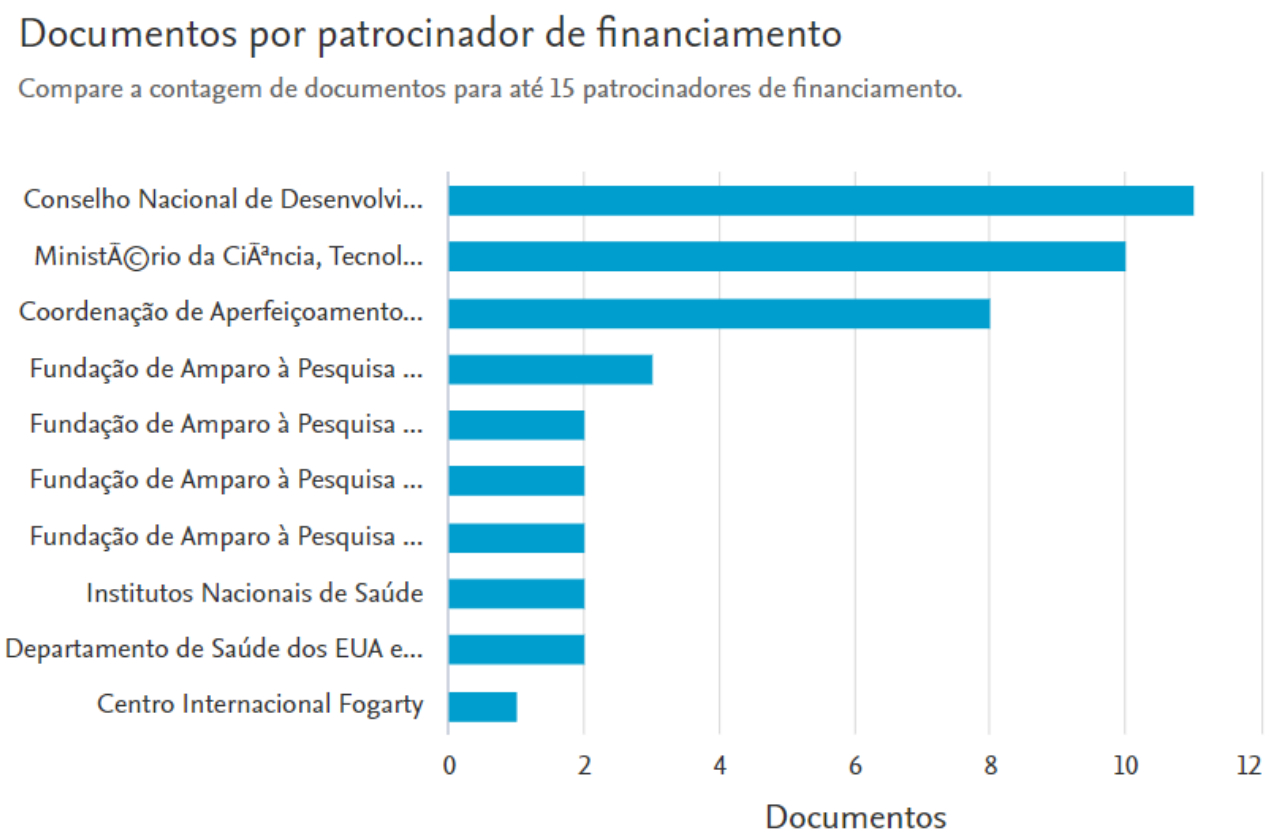

Fonte: Relatório de bibliometria da base Scopus (2021)

Criado em 1.951 pela Lei N. 1.310, o Conselho Nacional de Desenvolvimento Científico e Tecnológico é responsável pelo fomento de estudos tecnológicos e científicos brasileiros, bem como pela formação de cientistas no país (FARIA, 2021). Entre os serviços que o CNPq oferece, se encontram as bolsas de incentivo à pesquisa, que podem ser contempladas a alunos de graduação 
i.1] | Lucas Capita Quarto, Daniel Madeira Cardoso, Cristina de Fátima de Oliveira Brum Augusto de Souza e pós-graduação. Na contemporaneidade, o CNPq é considerado a maior agência de fomento do Brasil (FARIA, 2021).

\section{Análise dos documentos por afiliação (instituição)}

A produção científica brasileira a respeito da saúde ocupacional e o trabalhador rural representa $8,3 \%$ da produção científica mundial. Essas pesquisas estão vinculadas à 33 instituições brasileiras, com destaque para: Fundação Oswaldo Cruz (24 documentos); Universidade de São Paulo (14); Universidade Federal de Minas Gerais (10); Universidade Federal do Rio de Janeiro (8); Universidade Federal do Rio Grande (8) e a Universidade Estadual Paulista (8) (Figura 10).

Figura 10 - Documentos por agência de fomento

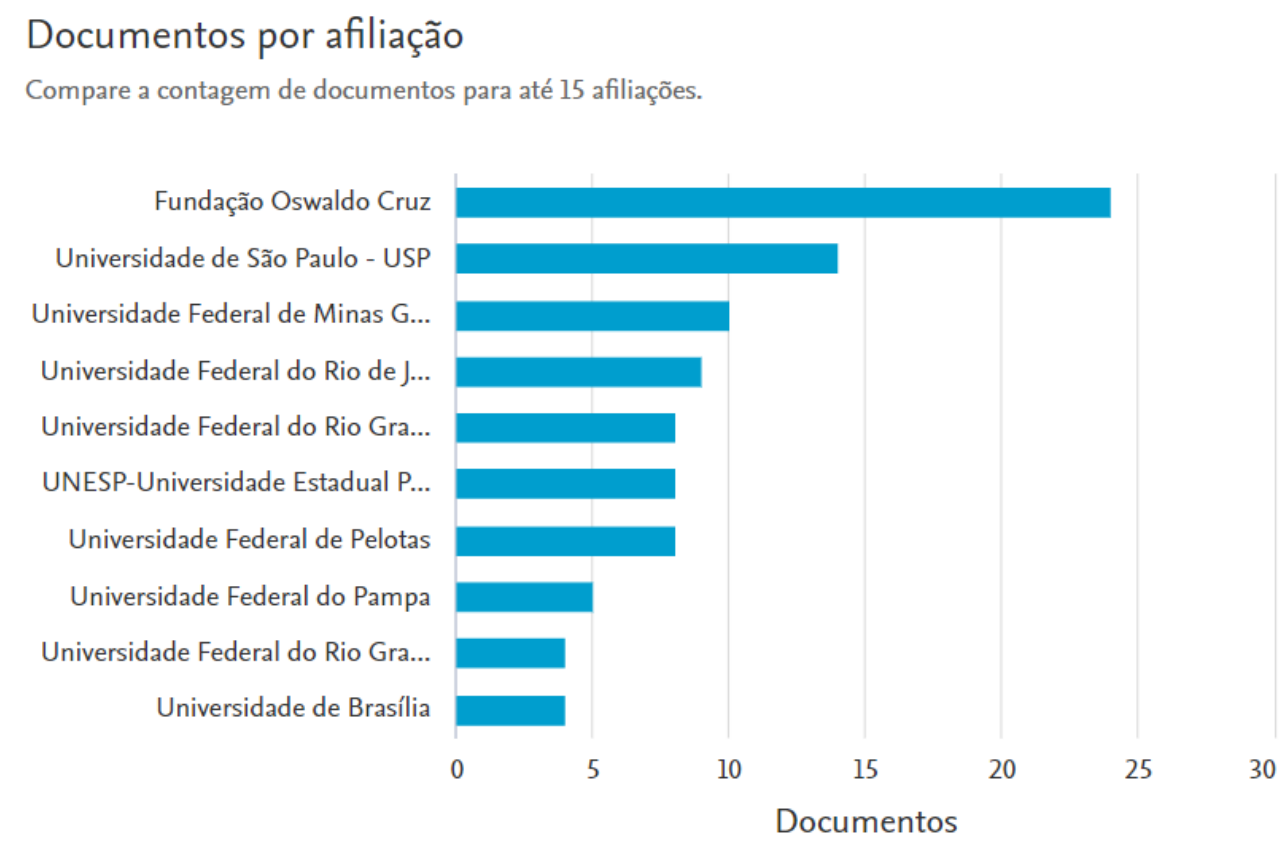

Fonte: Relatório de bibliometria da base Scopus (2021)

Localizada no Rio de Janeiro, a Fundação Oswaldo Cruz é uma agência vinculada ao Ministério da Saúde, considerada uma das melhores instituições de ciência e tecnologia da América Latina (FIOCRUZ, 2021). O objetivo Fiocruz é promover o desenvolvimento social, a saúde, gerar e difundir o conhecimento tecnológico e científico.

\section{Análise dos documentos por modalidade de divulgação}

Além de identificar os países e áreas do conhecimento que mais investem em pesquisas, as instituições mais produtivas e outros contextos, os estudos bibliométricos ainda possibilitam conhecer as modalidades de publicação (CARDOSO et al., 2020; QUARTO et al., 2020). 
Figura 11 - Documentos por modalidade de divulgação

\section{Documentos por tipo}

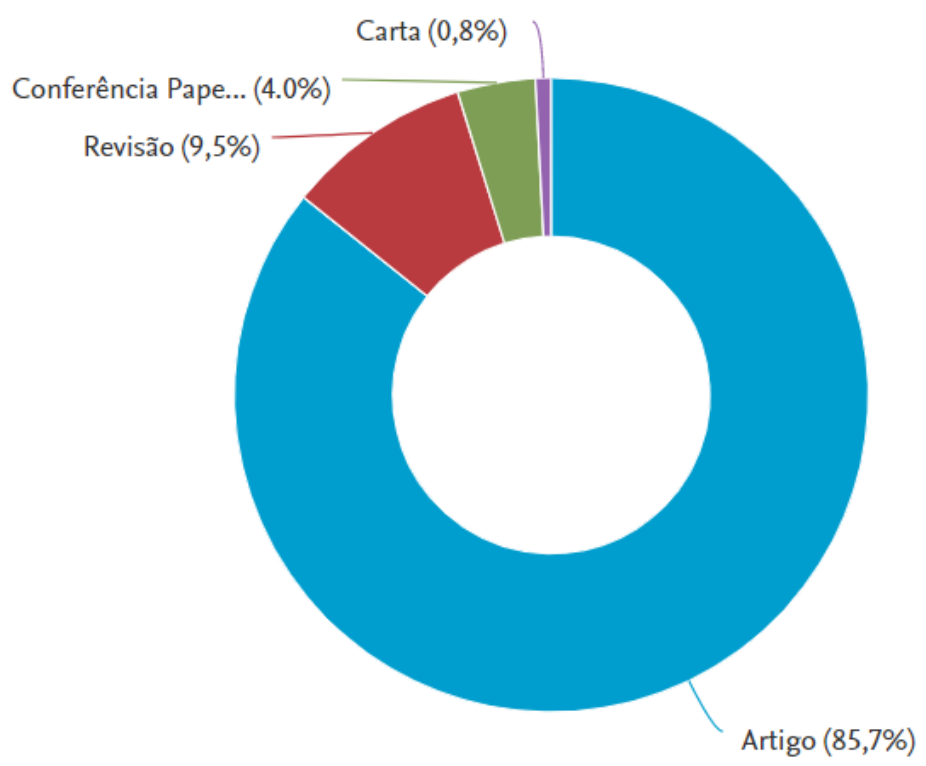

Fonte: Relatório de bibliometria da base Scopus (2021)

Conforme a Figura 11, o artigo científico é a modalidade de divulgação mais utilizada no Brasil para disseminar as pesquisas científicas a respeito da saúde do trabalhador rural. Em relação a esse tipo de documento, Quarto et al. (2020) mencionam que o artigo científico é a modalidade mais comum de divulgação de pesquisas científicas na contemporaneidade.

\section{CONCLUSÃO}

A presente pesquisa destaca a necessidade das pesquisas brasileiras se aterem às intervenções que precisam ser realizadas na esfera da saúde ocupacional do trabalhador rural para contribuir com o acervo científico sobre o assunto. Os resultados aqui obtidos corroboram os de pesquisas anteriores e demonstram que é fundamental incentivar o conhecimento acerca da saúde ocupacional do trabalhador rural.

\section{REFERÊNCIAS}

BEVILAQUA, M. D. et al. Implicação à saúde do trabalhador rural devido a exposições e uso de agrotóxicos: perspectivas para a enfermagem. Anuário Pesquisa e Extensão Unoesc, São Miguel do Oeste, v. 5, 2020.

CHUEKE, G. V.; AMATUCCI, M. O que é bibliometria? Uma introdução ao Fórum. Revista Eletrônica de Negócios Internacionais, São Paulo, v.10, n. 2, p. 1-5, 2015.

CSC. Revista da Associação Brasileira de Saúde Coletiva. About. Disponível em: < História da Revista - Revista Ciência \& Saúde Coletiva (cienciaesaudecoletiva.com.br)> Acessado em 14 de março de 2021. 
i.1. I Lucas Capita Quarto, Daniel Madeira Cardoso, Cristina de Fátima de Oliveira Brum Augusto de Souza

CSP. Caderno de Saúde Pública. About. Disponível em: < Cad. Saúde Pública- About the journal (scielo.br)> Acesso em: 14 mar. de 2021.

FARIA. C. Conselho Nacional de Desenvolvimento Científico e Tecnológico. CNPq.

Disponível em: < CNPq - InfoEscola> Acesso em: 16 mar. 2021.

FIOCRUZ. Fundação Oswaldo Cruz. A fundação. Acesso em: 18 mar. 2021. Disponível em: < Fiocruz - A Fundação - SUS >

OLIVEIRA, F. M. et al. Sistemas colaborativos; um estudo bibliométrico no período de 2015 a 2018. Interscienceplace, v.14, n.4, p. 192-240, 2019.

MARTINS, A. J.; FERREIRA, N. S. A ergonomia no trabalho rural. Rev. Eletrônica Atualiza Saúde. Salvador, v.2, n.2. Jul / dez, 2015.

MOREIRA, J.P. L. A saúde dos trabalhadores da atividade rural no Brasil. Cad. Saúde Pública, Rio de Janeiro, v. 31, n.8, p. 1698-1708, 2015.

QUARTO, L. C.; CARDOSO, D. M.; MANHÃES, F. C. Panorama Das Pesquisas Sobre Saúde E Segurança Do Trabalho. In: Pesquisas Interdisciplinares nas Engenharias. QUARTO, L. C.; SOUZA, S. M. F.; MANHÃES, F. C. (Org). Piracanjuba: Editora Conhecimento Livre, 2020.

RIBEIRO, H. C. M. Bibliometria: quinze anos de análise da produção acadêmica em periódicos brasileiros. Revista de Bibliotecnologia e Ciências da Informação, Monte Verde, v. 1, n. 69, 2017.

RSP. Revista de Saúde Pública. About. Disponível em: < Revista de Saúde Pública | A Revista - (usp.br)> Acessado em 14 de março de 2021.

Submetido em: mai. 2021.

Aprovado em: jun. 2021.

Publicado em: jun. 2021. 